\title{
De novo POGZ mutations are associated with neurodevelopmental disorders and microcephaly
}

\author{
Yizhou Ye, ${ }^{1}$ Megan T. Cho, ${ }^{1}$ Kyle Retterer, ${ }^{1}$ Nora Alexander, ${ }^{1}$ Tawfeg Ben-Omran, ${ }^{2}$ \\ Mariam Al-Mureikhi, ${ }^{2}$ Ingrid Cristian, ${ }^{3}$ Patricia G. Wheeler, ${ }^{3}$ Carrie Crain, ${ }^{3}$ \\ Dina Zand, ${ }^{4}$ Veronique Weinstein, ${ }^{4}$ Hilary J. Vernon, ${ }^{5}$ Rebecca McClellan, ${ }^{5}$ \\ Vidya Krishnamurthy, ${ }^{6}$ Patrik Vitazka, ${ }^{1}$ Francisca Millan, ${ }^{1}$ and Wendy K. Chung ${ }^{7}$ \\ ${ }^{1}$ GeneDx, Gaithersburg, Maryland 20877, USA; ${ }^{2}$ Clinical and Metabolic Genetics, Hamad Medical \\ Corporation, Doha, Qatar; ${ }^{3}$ Nemours Children's Hospital, Orlando, Florida 32827, USA; ${ }^{4}$ Children's National \\ Medical Center, Washington, D.C. 20010, USA; ${ }^{5}$ Kennedy Krieger Institute, Baltimore, Maryland 21205, USA; \\ ${ }^{6}$ Pediatrics and Genetics, Alpharetta, Georgia 30005, USA; ${ }^{7}$ Departments of Pediatrics and Medicine, \\ Columbia University Medical Center, New York, New York 10032, USA
}

Abstract Seven patients with similar phenotypes of developmental delay and microcephaly were found by whole-exome sequencing to have de novo loss-of-function mutations in POGZ. POGZ is a pogo transposable element-derived protein with a zinc finger cluster. The protein is involved in normal kinetochore assembly and mitotic sister chromatid cohesion and mitotic chromosome segregation. POGZ deficiency may affect mitosis, disrupting brain development and function.

[Supplemental material is available for this article.]

\section{INTRODUCTION}

Corresponding author:wkc15@ columbia.edu

(C) 2015 Ye et al. This article is distributed under the terms of the Creative Commons

Attribution-NonCommercial License, which permits reuse and redistribution, except for commercial purposes, provided that the original author and source are credited.

Ontology terms: intellectual disability, severe; microcephaly; severe global developmental delay

Published by Cold Spring Harbor Laboratory Press

doi: $10.1101 /$ mcs.a000455
De novo mutations are increasingly recognized to play an important role in sporadic, severe neurodevelopmental disorders because of the deleterious effect on reproductive fitness (Veltman and Brunner 2012). For genetically heterogeneous disorders such as developmental delay and intellectual disability (ID), whole-exome sequencing (WES) can identify a genetic etiology in up to $30 \%$ of cases when chromosomal array and fragile $X$ testing are normal (Yang et al. 2013, 2014). When analyzed together with parental samples, WES readily identifies de novo variants. Here we describe seven patients with a similar phenotype of developmental delay and microcephaly, six of whom have de novo predicted loss-of-function mutations in pogo transposable element with ZNF domain (POGZ). POGZ is located on Chromosome 1q21.3 and is a transposable element with a zinc finger domain involved in kinetochore assembly and mitotic sister chromatid cohesion and mitotic chromosome segregation (Nozawa et al. 2010).

\section{RESULTS}

For 2415 patients referred for clinical WES for neurodevelopmental disorders, exome sequencing produced an average of $\sim 9.0 \mathrm{~Gb}$ of sequence per sample. Mean coverage of captured regions was $\sim 96 \times$ per sample with $>96 \%$ covered with at least $10 \times$ coverage, an 
Table 1. Variants identified from whole-exome sequencing of five families

\begin{tabular}{lccc}
\hline & $\begin{array}{c}\text { Filtering } \\
\text { results }\end{array}$ & $\begin{array}{c}\text { Manual } \\
\text { review }\end{array}$ & $\begin{array}{c}\text { Resulting genes of } \\
\text { interest }\end{array}$ \\
\hline $\begin{array}{l}\text { Homozygous (\# seq changes) } \\
\begin{array}{l}\text { Compound Heterozygous (\# seq } \\
\text { changes) }\end{array}\end{array}$ & $74(77)$ & $0(0)$ & $0(0)$ \\
$\begin{array}{l}\text { De novo (\# seq changes) } \\
\text { X-linked genes (\# seq changes) }\end{array}$ & $93(85)$ & $1(2)$ & $0(0)$ \\
Total genes (\# seq changes) & $32(32)$ & $3(3)$ & $1(1)$ \\
\hline
\end{tabular}

average of $94 \%$ of base call quality of Q30 or greater, and an overall average mean quality score of >Q36 (Supplemental Table S1). Filtering of common SNPs (>10\% frequency present in 1000 Genomes database) resulted in approximately 5500 variants per proband sample. Seven unrelated patients were found to have heterozygous changes in POGZ that are predicted to be deleterious, $0.29 \%$ of the cases referred for neurodevelopmental disorders. In these families, 230 genes (289 unique sequence changes) of interest were identified across five families when considering all possible modes of inheritance (Table 1). Because parental samples were not available for Patient 5 , inheritance of variants could not be determined, so those data are not included in Table 1. Data from Patient 6 were not included in Table 1 because the heterozygous loss of function of POGZ was the result of a copy-number variant (Retterer et al. 2014). Evaluation of the 230 genes eliminated 229 genes that did not segregate with the phenotype in the family, leaving one gene for which there was clinical overlap of the patients' phenotype.

The seven patients in this report all have novel variants predicted to cause loss of function including frameshift and nonsense mutations and one patient with a heterozygous deletion of exons 4-19 (Fig. 1). In six patients the POGZ variant was de novo while the inheritance could not be determined in the seventh patient because parental samples were not available for testing. None of these variants are observed in approximately 6500 individuals of European and African American ancestry in the NHLBI Exome Sequencing Project, in ExAC, or in our own local database of 8800 exomes from unrelated unaffected adults, indicating that these variants are not common. In the six patients for whom parents were available, these variants are de novo. In addition, Individual 3 was found to be hemizygous for an inherited variant in the ZNF81 gene, located on the X chromosome. Although this gene may be associated with ID based on one missense variant reported previously, variants in this gene have also been frequently seen in the NHLBI Exome Sequencing Project; therefore, it is more likely that the POGZ variant explains the ID in this individual, although the ZNF81 variant cannot be completely ruled out. Individual 6 has a deletion involving exons 4 through 19 of POGZ, and it also encompasses part of the PSMB4 gene that has not previously been associated with any phenotypic consequences.

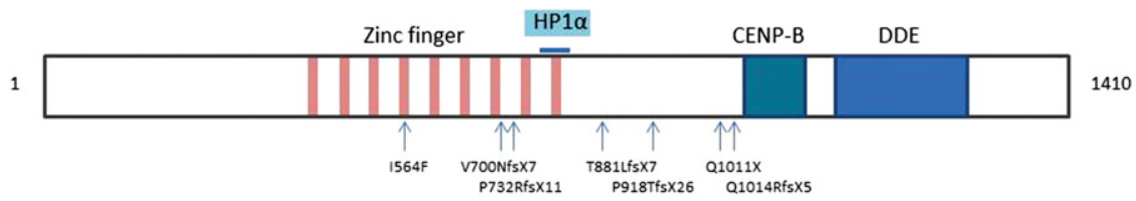

CENP-B: a centromere protein (CENP)-B-like DNA binding domain;

DDE domain: originated from a transposase encoded by a pogo-like DNA transposon

Figure 1. Location of pathogenic variants in POGZ. 
COLD SPRING HARBOR Molecular Case Studies
POGZ mutations cause neurodevelopmental delay and microcephaly
The phenotype of the seven unrelated patients with predicted loss of function rare variants in POGZ that are predicted to be deleterious are similar (Table 2). The patients range in age from 1 to $16 \mathrm{yr}$ old. All seven patients are developmentally delayed including significantly delayed walking and talking. Six patients whose cognitive status was known have intellectual disabilities based on clinician report from standardized measures such as developmental or intelligence quotients. Head circumferences ranged from $<3$ percentile to 25 percentile, and three patients are microcephalic, with an additional four patients with head circumferences at the 3 percentile to 25 percentile. Six patients whose muscle tone is known are hypotonic. Six patients have dysmorphic facial features, including hypertelorism and bushy eyebrows as well as some patients who were noted to have a tented upper lip, short philtrum, and abnormal nose (underdeveloped alae, short nose, broad nasal bridge) (Fig. 2). A distinguishing physical feature is the hands with broad and/or adducted thumbs and syndactyly, which is present in five patients. Three out of five patients who had brain MRIs were found to have abnormalities. One patient has polymicrogyria, one has a small optic chiasm, dysplasia of cerebellum, and periventricular white matter injury, and another has cortical atrophy and atrophy of the corpus callosum. Three patients have cyclic vomiting. Less commonly reported clinical features include behavioral abnormalities (autism, anxiety, and attention deficit hyperactivity disorders), contractures, failure to thrive, and migraines.

\section{DISCUSSION}

Patients from seven independent families with a similar phenotype of severe developmental delay and small head size were all found to have likely gene damaging mutations in POGZ that were de novo in all six cases evaluable.

POGZ is essential for normal kinetochore assembly, mitotic sister chromatid cohesion and mitotic chromosome segregation (Nozawa et al. 2010). POGZ modulates mitotic progression at multiple steps, and most of its regulatory roles are tightly linked to the chromosomal passenger complex (CPC) (Nozawa et al. 2010). CPC is composed of four different subunits, including Aurora B that regulates key events during mitosis (Carmena et al. 2012). It is dynamically localized at different times during mitosis to ensure the effective and spatially restricted phosphorylation of substrates (Carmena et al. 2012). During interphase, CPC targets heterochromatin protein 1 a (HP1 $\alpha$ ), which interacts with chromatin, maintains heterochromatin structure, and is localized to pericentromeric heterochromatin and chromosome arms (Yamagishi et al. 2008; Prasanth et al. 2010; Carmena et al. 2012). When entering mitosis, HP1 $\alpha$ and CPC are dissociated from the chromosome arms. POGZ, competing with PxVxL-domain proteins, binds to HP1a. When POGZ binds HP1a, the HP1a-chromatin interaction becomes unstable. Aurora B-dependent phosphorylation at Ser 10 of histone H3 promotes the dissociation of HP1a from the chromosome arms (Fischle et al. 2005; Hirota et al. 2005). Both Aurora B activation and POGZ binding contribute to the dissociation of HP1 a from the chromatin, as well as the facilitation of CPC enrichment at inner centromeres. In late mitosis, CPC binds to microtubules at the spindle midzone and executes anaphase chromatid compaction (Carmena et al. 2012).

POGZ-knockdown cells exhibit disruption of HP1a dissociation from the chromosome arms, and as a consequence, disrupted CPC translocalization. POGZ depletion also reduced the phosphorylation activity of Aurora B kinase (Nozawa et al. 2010). Similar to observations of POGZ depleted cells, Aurora B inhibition impairs CPC localization at centromeres during mitosis (Nozawa et al. 2010).

As a result of POGZ deficiency, the majority of cells is unable to form metaphase plates, and will exit mitosis prematurely. One of the characteristic features of premature mitotic exit is the formation of polyploidy cells, which can lead to cell death or genome instability in 


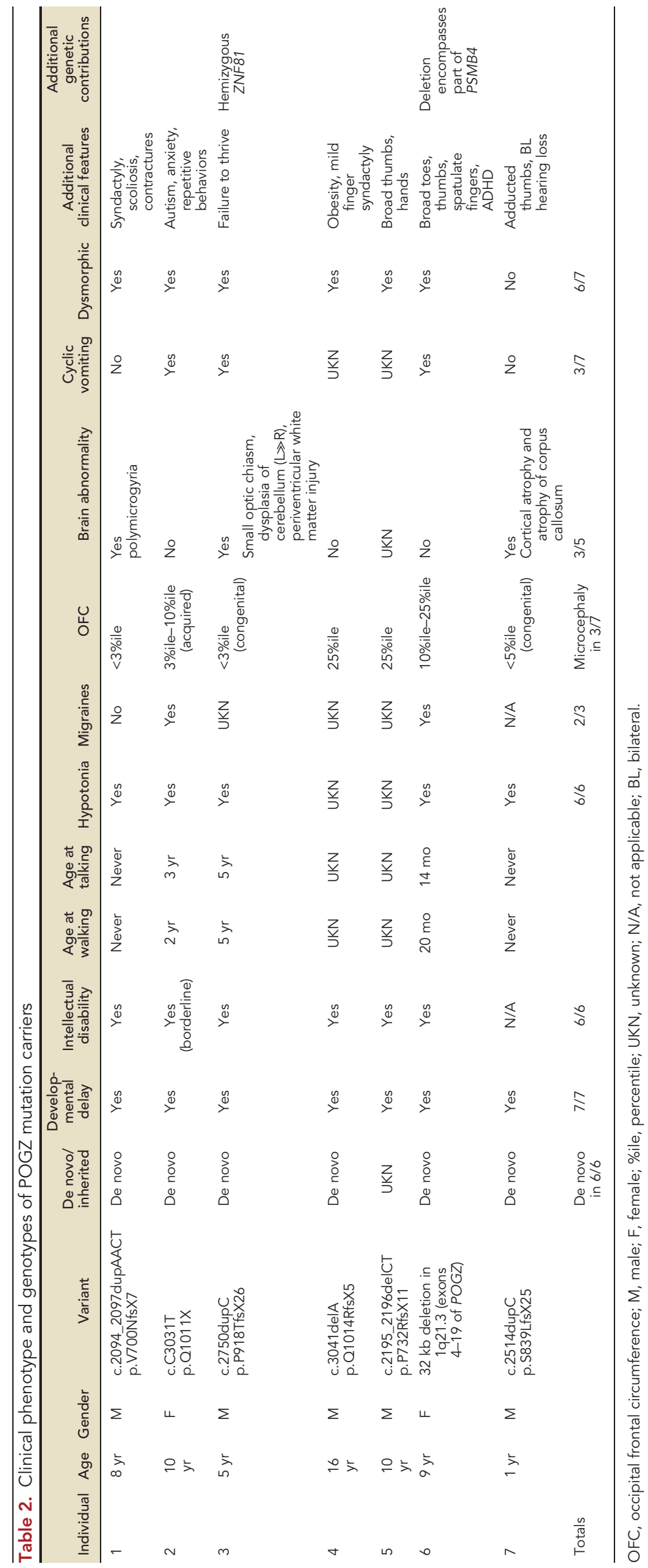


COLD SPRING HARBOR Molecular Case Studies

POGZ mutations cause neurodevelopmental delay and microcephaly

Individual 1
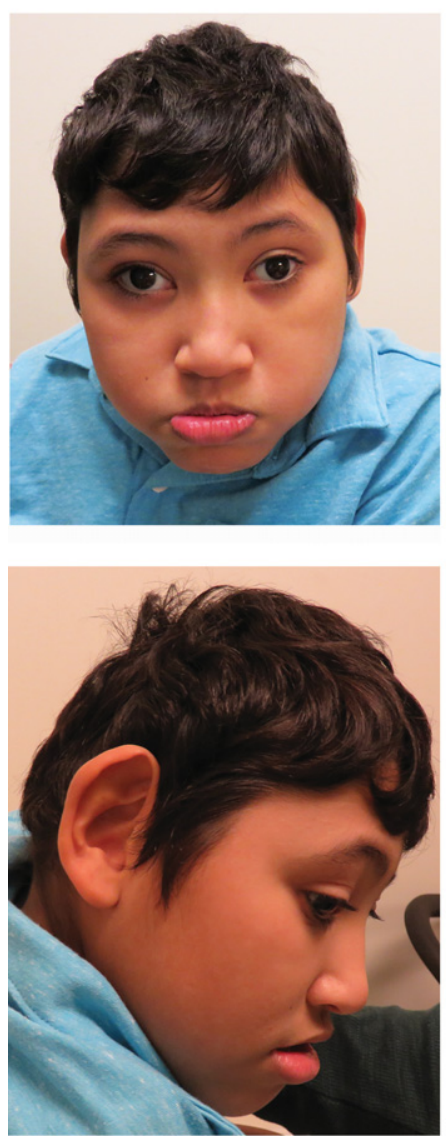

Individual 3
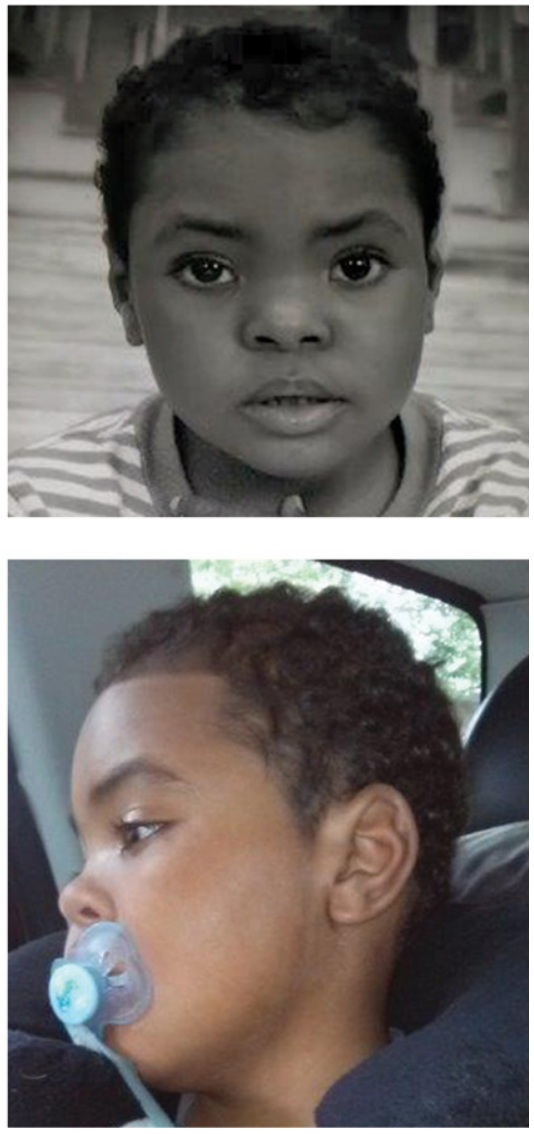

Figure 2. Facial characteristics of two individuals with a de novo POGZ variant. Individual 1: large ears, hypertelorism, oral hypotonia, full lower lip. Individual 3: hypertelorism, depressed and broad nasal bridge, and tented upper lip.

subsequent division cycles. The POGZ deficiency phenotype is similar to that of loss-of-function mutation models of other CPC proteins: the mutant cells undergo multiple consecutive abnormal mitoses, producing polyploidy cells, which results in abnormal organ development (Hanson et al. 2005; Thornton and Woods 2009). Mutations in genes, such as ASPM, that lead to defects in the mitotic spindle, cause congenital microcephaly and intellectual disability (Bond et al. 2002, 2003; Desir et al. 2008). During prenatal development, haploinsufficiency of POGZ would be predicted to lead to depletion of neurogenic progeny, limiting the total number of neurons and resulting in microcephaly. Two of the patients had documented congenital microcephaly to support this mechanism.

All mutations in our series are predicted to act as loss-of-function alleles and result in haploinsufficiency, either through introducing premature stop codons or abnormal splicing leading to nonsense-mediated mRNA decay. De novo loss of function mutations in POGZ have been identified in individuals with autism spectrum disorder, intellectual disability, and schizophrenia (lossifov et al. 2012; Neale et al. 2012; Fromer et al. 2014; Gilissen et al. 2014; Deciphering Developmental Disorders Study 2015). Similar to other genes that produce neurodevelopmental disorders, there is a range of related neurobehavioral phenotypes associated with POGZ that includes intellectual disability, autism spectrum disorder, 
COLD SPRING HARBOR Molecular Case Studies
POGZ mutations cause neurodevelopmental delay and microcephaly and schizophrenia. The role of POGZ in mitosis suggests a possible role in regulating neuronal proliferation and could explain why patients with POGZ mutations are microcephalic and dysmorphic, including anomalies with craniofacial and limb development.

\section{METHODS}

Genomic DNA was extracted from whole blood from the affected children and their parents. Exome sequencing was performed on exon targets isolated by capture using the Agilent SureSelect Human All Exon V4 (50-Mb) kit or the Clinical Research Exome (Agilent Technologies). One microgram of DNA from blood specimen was sheared into 350-400bp fragments, which were then repaired, ligated to adaptors, and purified for subsequent PCR amplification. Amplified products were then captured by biotinylated RNA library baits in solution following the manufacturer's instructions. Bound DNA was isolated with streptavidin-coated beads and reamplified. The final isolated products were sequenced using the Illumina HiSeq 2000 or 2500 sequencing system with 100-bp paired-end reads (Illumina). DNA sequence was mapped to the published human genome build UCSC hg19/GRCh37 reference sequence using BWA with the latest internally validated version at the time of sequencing, progressing from BWA v0.5.8 through BWA-MEM v0.7.8 (Li and Durbin 2009; Li 2012). Targeted coding exons and splice junctions of known proteincoding RefSeq genes were assessed for average depth of coverage with a minimum depth of $10 \times$ required for inclusion in downstream analysis. Local realignment around insertiondeletion sites was performed using the Genome Analysis Toolkit v1.6 (DePristo et al. 2011). Variant calls were generated simultaneously on all sequenced family members using SAMtools v0.1.18 ( $\mathrm{Li}$ et al. 2009). All coding exons and surrounding intron/exon boundaries were analyzed. Automated filtering removed common sequence changes (defined as $>10 \%$ frequency present in 1000 Genomes database). The targeted coding exons and splice junctions of the known protein-coding RefSeq genes were assessed for the average depth of coverage and data quality threshold values. Copy number variants were also called directly from the WES data (Retterer et al. 2014). Whole-exome sequence data for all sequenced family members was analyzed using GeneDx's XomeAnalyzer (a variant annotation, filtering, and viewing interface for WES data), which includes nucleotide and amino acid annotations, population frequencies (NHLBI Exome Variant Server and 1000 Genomes databases), in silico prediction tools, amino acid conservation scores, and mutation references. Variants were filtered based on inheritance patterns, gene lists of interest, phenotype, and population frequencies, as appropriate. Resources including the Human Gene Mutation Database (HGMD), 1000 Genomes database, NHLBI Exome Variant Server, OMIM, PubMed, ClinVar, and Exome Aggregation Consortium (ExAC; URL: http://exac.broadinstitute.org) were used to evaluate genes and detected sequence changes of interest. The general assertion criteria for variant classification are publicly available on the GeneDx ClinVar submission page (http://www.ncbi.nlm.nih.gov/clinvar/submitters/26957/). Additional searches were performed using specific gene lists related to ID. Identified sequence changes of interest were confirmed in all members of the trio by conventional di-deoxy DNA sequence analysis using an ABI 3730 (Life Technologies) and standard protocols with a new DNA preparation.

\section{ADDITIONAL INFORMATION}

\section{Ethics Statement}

The study was approved by the Institutional Review Board of Columbia University and written consent was obtained for collecting blood samples and sequencing from all study participants. 
COLD SPRING HARBOR Molecular Case Studies
POGZ mutations cause neurodevelopmental delay and microcephaly
Competing Interest Statement

Y.Y., M.T.C., K.R., N.A., P.V., and F.M. are employees of GeneDx. W.K.C. is a consultant to

BioReference Laboratories.

Received June 3, 2015; accepted in revised form July 28, 2015.

\section{Database Deposition and Access}

Whole-exome sequencing data are not publicly available because patient consent could not be obtained. These POGZ variants have been deposited in ClinVar (http://www. ncbi.nlm.nih.gov/clinvar/) under accession numbers SCV000240171 and SCV000240173SCV000240178.

\section{Acknowledgments}

We thank the families for their generous contribution.

\section{Author Contributions}

Y.Y. analyzed the data, drafted and critically reviewed the manuscript. M.T.C. analyzed the data and critically reviewed the manuscript. K.R. generated and analyzed the data and critically reviewed the manuscript. N.A. analyzed the data and critically reviewed the manuscript. T.B.-O. provided the clinical data and critically reviewed the manuscript. M.A.-M. provided the clinical data and critically reviewed the manuscript. I.C. provided the clinical data and critically reviewed the manuscript. P.G.W. provided the clinical data and critically reviewed the manuscript. C.C. provided the clinical data and critically reviewed the manuscript. D.Z. provided the clinical data and critically reviewed the manuscript. V.W. provided the clinical data and critically reviewed the manuscript. H.J.V. provided the clinical data and critically reviewed the manuscript. R.M. provided the clinical data and critically reviewed the manuscript. V.K. provided the clinical data and critically reviewed the manuscript. F.M. analyzed the data and critically reviewed the manuscript. W.K.C. conceived of the study, analyzed the data, drafted and critically reviewed the manuscript. P.V. conceived of the study, analyzed the data, drafted and critically reviewed the manuscript.

\section{REFERENCES}

Bond J, Roberts E, Mochida GH, Hampshire DJ, Scott S, Askham JM, Springell K, Mahadevan M, Crow YJ, Markham AF, et al. 2002. ASPM is a major determinant of cerebral cortical size. Nat Genet 32: $316-$ 320.

Bond J, Scott S, Hampshire DJ, Springell K, Corry P, Abramowicz MJ, Mochida GH, Hennekam RC, Maher ER, Fryns JP, et al. 2003. Protein-truncating mutations in ASPM cause variable reduction in brain size. Am J Hum Genet 73: 1170-1177.

Carmena M, Wheelock M, Funabiki H, Earnshaw WC. 2012. The chromosomal passenger complex (CPC): from easy rider to the godfather of mitosis. Nat Rev Mol Cell Biol 13: 789-803.

DePristo MA, Banks E, Poplin R, Garimella KV, Maguire JR, Hartl C, Philippakis AA, del Angel G, Rivas MA, Hanna $M$, et al. 2011. A framework for variation discovery and genotyping using next-generation DNA sequencing data. Nat Genet 43: 491-498.

Desir J, Cassart M, David P, Van Bogaert P, Abramowicz M. 2008. Primary microcephaly with ASPM mutation shows simplified cortical gyration with antero-posterior gradient pre- and post-natally. Am J Med Genet A 146A: 1439-1443.

Deciphering Developmental Disorders Study. 2015. Large-scale discovery of novel genetic causes of developmental disorders. Nature 519: 223-228.

Fischle W, Tseng BS, Dormann HL, Ueberheide BM, Garcia BA, Shabanowitz J, Hunt DF, Funabiki H, Allis CD. 2005. Regulation of HP1-chromatin binding by histone H3 methylation and phosphorylation. Nature 438: 1116-1122.

Fromer M, Kavanagh DH, Williams HJ, DwyerS, Gormley P, Georgieva L, Rees E, Palta P, Ruderfer DM, Carrera N, et al. 2014. De novo mutations in schizophrenia implicate synaptic networks. Nature 506: 179-184.

Gilissen C, Hehir-Kwa JY, Thung DT, van de Vorst M, van Bon BW, Willemsen MH, Kwint M, Janssen IM, Hoischen A, Schenck A, et al. 2014. Genome sequencing identifies major causes of severe intellectual disability. Nature 511: 344-347.

Hanson KK, Kelley AC, Bienz M. 2005. Loss of Drosophila borealin causes polyploidy, delayed apoptosis and abnormal tissue development. Development 132: 4777-4787. 
C OLD SPRING HARBOR Molecular Case Studies
POGZ mutations cause neurodevelopmental delay and microcephaly

Hirota T, Lipp JJ, Toh BH, Peters JM. 2005. Histone H3 serine 10 phosphorylation by Aurora B causes HP1 dissociation from heterochromatin. Nature 438: 1176-1180.

lossifov I, Ronemus M, Levy D, Wang Z, Hakker I, Rosenbaum J, Yamrom B, Lee YH, Narzisi G, Leotta A, et al. 2012. De novo gene disruptions in children on the autistic spectrum. Neuron 74: 285-299.

Li H. 2012. Exploring single-sample SNP and INDEL calling with whole-genome de novo assembly. Bioinformatics 28: 1838-1844.

Li H, Durbin R. 2009. Fast and accurate short read alignment with Burrows-Wheeler transform. Bioinformatics 25: $1754-1760$.

Li H, Handsaker B, Wysoker A, Fennell T, Ruan J, Homer N, Marth G, Abecasis G, Durbin R; 1000 Genome Project Data Processing Subgroup. 2009. The Sequence Alignment/Map format and SAMtools. Bioinformatics 25: 2078-2079.

Neale BM, Kou Y, Liu L, Ma'ayan A, Samocha KE, Sabo A, Lin CF, Stevens C, Wang LS, Makarov V, et al. 2012. Patterns and rates of exonic de novo mutations in autism spectrum disorders. Nature 485: 242-245.

Nozawa RS, Nagao K, Masuda HT, Iwasaki O, Hirota T, Nozaki N, Kimura H, Obuse C. 2010. Human POGZ modulates dissociation of HP1 a from mitotic chromosome arms. Nat Cell Biol 12: 719-727.

Prasanth SG, Shen Z, Prasanth KV, Stillman B. 2010. Human origin recognition complex is essential for HP1 binding to chromatin and heterochromatin organization. Proc Natl Acad Sci 107: 15093-15098.

Retterer K, Scuffins J, Schmidt D, Lewis R, Pineda-Alvarez D, Stafford A, Schmidt L, Warren S, Gibellini F, Kondakova A, et al. 2014. Assessing copy number from exome sequencing and exome array CGH based on CNV spectrum in a large clinical cohort. Genet Med. doi: 10.1038/gim.2014.160.

Thornton GK, Woods CG. 2009. Primary microcephaly: do all roads lead to Rome? Trends Genet 25: 501-510.

Veltman JA, Brunner HG. 2012. De novo mutations in human genetic disease. Nat Rev Genet 13: 565-575.

Yamagishi Y, Sakuno T, Shimura M, Watanabe Y. 2008. Heterochromatin links to centromeric protection by recruiting shugoshin. Nature 455: 251-255.

Yang Y, Muzny DM, Reid JG, Bainbridge MN, Willis A, Ward PA, Braxton A, Beuten J, Xia F, Niu Z, et al. 2013. Clinical whole-exome sequencing for the diagnosis of mendelian disorders. $N$ Engl J Med 369: 1502-1511.

Yang Y, Muzny DM, Xia F, Niu Z, Person R, Ding Y, Ward P, Braxton A, Wang M, Buhay C, et al. 2014. Molecular findings among patients referred for clinical whole-exome sequencing. JAMA 312: 1870-1879. 


\section{COLD SPRING HARBOR Molecular Case Studies}

\section{De novo POGZ mutations are associated with neurodevelopmental disorders and microcephaly}

Yizhou Ye, Megan T. Cho, Kyle Retterer, et al.

Cold Spring Harb Mol Case Stud 2015, 1: a000455

Access the most recent version at doi: $10.1101 / \mathrm{mcs} .0000455$

$\underset{\text { Material }}{\operatorname{Supplementary}} \quad{ }_{1}^{\text {http://molecularcasestudies.cshlp.org/content/suppl/2015/09/03/1.1.a000455.DC }}$

References This article cites 24 articles, 2 of which can be accessed free at: http://molecularcasestudies.cshlp.org/content/1/1/a000455.full.html\#ref-list-1

License This article is distributed under the terms of the Creative Commons Attribution-NonCommercial License, which permits reuse and redistribution, except for commercial purposes, provided that the original author and source are credited.

Email Alerting Receive free email alerts when new articles cite this article - sign up in the box at the Service top right corner of the article or click here. 